\title{
REVISTA
}

Revista Educación

ISSN: 0379-7082

ISSN: 2215-2644

revedu@gmail.com

Universidad de Costa Rica

Costa Rica

\section{Dar voz a los protagonistas: La participación estudiantil en el proceso de enseñanza-aprendizaje}

Hidalgo, Nina; Perines, Haylen

Dar voz a los protagonistas: La participación estudiantil en el proceso de enseñanza-aprendizaje

Revista Educación, vol. 42, núm. 2, 2018

Universidad de Costa Rica, Costa Rica

Disponible en: http://www.redalyc.org/articulo.oa?id=44055139030

DOI: https://doi.org/10.15517/revedu.v42i2.27567

Esta obra está bajo una Licencia Creative Commons Atribución-NoComercial-SinDerivar 3.0 Internacional. 
Dar voz a los protagonistas: La participación estudiantil en el proceso de enseñanzaaprendizaje

Give Voice to the Participants: Student Participation in the Teaching-Learning Process

Nina Hidalgo [1]

Universidad Autónoma de Madrid, España

nina.hidalgo@uam.es

Haylen Perines [2]

Universidad Autónoma de Madrid, España

profesorahaylen@gmail.com
DOI: https://doi.org/10.15517/revedu.v42i2.27567

Redalyc: http://www.redalyc.org/articulo.oa?id=44055139030

Recepción: 23 Diciembre 2016

Aprobación: 16 Abril 2018

\section{RESUMEN:}

El objetivo principal de este artículo es describir las prácticas pedagógicas y las actitudes que docentes tienen hacia la participación de estudiantes. Para ello, se ha desarrollado un estudio ex post facto descriptivo con 177 docentes pertenecientes a centros de Educación Secundaria de cuatro comunidades autónomas de España. La investigación se centra en tres aspectos de las prácticas de participación que desarrollan el personal docente: la selección del contenido a enseñar, las estrategias didácticas a llevar a cabo y la evaluación de los aprendizajes. Los resultados apuntan a que estos cuerpos permiten que sus estudiantes participen principalmente en la selección de las estrategias didácticas; pero muy poco en la evaluación o en la selección de los contenidos. Además, entre las tres prácticas de participación analizadas la opción más elegida por el profesorado es informar al alumnado con antelación de lo que se va a realizar. Por otro lado, muy poca cantidad de docentes afirma dar espacio al consenso o a la implicación de estos.

Palabras Clave: Participación estudiantil, aprendizaje, evaluación de la educación, docente.

\section{ABstract:}

The main objective of this research is to describe the pedagogical practices and the attitudes teachers have towards student participation. For this, an ex post facto descriptive study was developed with 177 teachers belonging to secondary education schools from four autonomous communities in Spain. The research focuses on three aspects of participation practices that teachers develop: selection of content to teach, teaching strategies to be carried out and learning assessment. The results suggest that teachers allow students to participate mainly in the selection of teaching strategies, but insufficient participation is allowed in the selection of assessments or contents. In addition, among the three participation practices analyzed, the most selected option by the teachers is to inform the students in advance about the subject matter to be carried out during the lesson. On the other hand, few teachers give space for consensus or student participation.

KEYWORDS: Student participation, learning, educational assessment, teacher.

\section{INTRODUCCIÓN}

Hace más de 20 años, el profesor Fernández Enguita emitía esta contundente frase que nos removía la conciencia y nos invitaba a reflexionar sobre las complejidades que subyacen al término participación en educación:

\section{Notas DE AUTOR}

[1] Máster en Calidad y Mejora de la Educación Personal Docente e Investigador en Formación, Universidad Autónoma de Madrid. Editora de la Revista Iberoamericana de Evaluación Educativa (RIEE) de la red RINACE y de la Revista Internacional de Educación para la Justicia Social (RIEJS). Miembro del comité evaluador de revistas educativas como REICE y RIEJS.

[2] Doctora en Educación. Personal investigador en formación, Universidad Autónoma de Madrid. Miembro del comité evaluador de revistas educativas como REICE y RIEJS. 
El término participación [en educación] es una muestra de lenguaje pervertido, presencia permanente en el discurso escolar oficial y oficioso que tiene que ver con cualquier cosa menos con la realidad, y quien crea poder conocer ésta a través de aquél no conseguirá sino engañarse. (Fernández Enguita, 1992, p. 80)

Los años pasan y el sistema educativo ha vivido innumerables cambios, evoluciones y progresos, sin embargo, ¿es posible afirmar que las palabras de Fernández Enguita siguen vigentes en la actualidad?

Respecto a la bibliografía en torno al tema de la participación, varios estudios coinciden en afirmar que, aunque en el discurso escolar se manifieste que el estudiantado participa en su proceso de enseñanzaaprendizaje, la realidad es que la escuela no le da una oportunidad real, convirtiéndolo en mero receptor (Bretones, 1996; Cook-Sather 2001, 2002; Fredricks, Blumenfeld y Paris, 2004; Jagersma y Parsons, 2011; Leithwood, Mulford y Silins, 2004; Mitra y Gross, 2009; Ros, 2009; San Fabián, 1994). El estudiantado es el "convidado de piedra" de la educación, como dice el propio Fernández Enguita (1992).

Hablar de participación no es un añadido, como si este término fuese un factor más de la educación. Por el contrario, es una de las claves de todo proceso educativo. En esta línea, Bernstein (1990) considera que son tres los derechos que tiene cada estudiante: el derecho al crecimiento social, intelectual y personal, el derecho de inclusión y el derecho a participar en las decisiones que le afectan. Igualmente, la participación se está convirtiendo en una de las bases de la justicia social (Fraser, 2008; Young, 2011), de tal forma que, junto con la redistribución y el reconocimiento (Murillo y Hernández-Castilla, 2011; 2014), forman el corpus tridimensional que define este concepto.

En coherencia con esta importancia, y acorde con la obsesión de los nuevos tiempos por el desempeño estudiantil, una de las líneas de investigación que está adquiriendo más fuerza en los últimos años es precisamente aquella que considera que la participación e implicación de estos grupos en el aula y en el proceso de enseñanza provoca un cambio significativo en su aprendizaje (Allwright, 1984; Morell, 2004; 2007). Tal como afirma Solana (2003), profundizar en los intereses, motivaciones y opiniones de nuestro alumnado nos ayuda a implicarle en el aula y mejorar conjuntamente el proceso de enseñanza-aprendizaje. Asimismo, Morell (2009, p.11) afirma que "como profesores, deberíamos pensar en las formas de hacer que nuestros alumnos realmente se involucren en las clases".

Este artículo pretende conocer las prácticas de participación que desarrollan docentes de educación secundaria. Para ello se ha realizado un estudio ex post facto, cuyo objetivo general ha sido describir las prácticas pedagógicas y las actitudes hacia la participación estudiantil de 177 docentes pertenecientes a centros de educación secundaria de cuatro comunidades autónomas de España.

\section{REVISIÓN BIBLIOGRÁFICA}

La principal característica del concepto de participación es la intervención de estudiantes en la toma de decisiones en el aula. Estas decisiones deben ir en consonancia con aquello que se ha dialogado y decidido de forma conjunta entre docentes y estudiantes (Bretones, 1996). A pesar de esta convicción, no es fácil definir con certeza el concepto. El mismo Bretones (1996) afirma que la "participación es uno de esos términos manidos, tan frecuentes en el discurso sobre la educación, que a fuerza de designarlo todo terminan por no significar nada" (p. 28).

El término participación es escurridizo, fragmentado y existe una extensa bibliografía en relación con sus distintos enfoques. Sin embargo, son tantos los significados que se han dado a esta palabra y desde campos tan diversos que resulta casi imposible llegar a un concepto unificado (San Fabián, 1994; Susinos y Ceballos, 2012).

Desde la perspectiva de Bretones (1996):

La participación es un continuo, se participa más o menos en una situación o en un proceso pero ello no impide considerar cuando se alcanza o no el nivel crítico que permita hacer una valoración aceptable o inaceptable. Además la participación exige repartir la capacidad de decisión, no es una cuestión de estar más o menos intrínsecamente motivado. Por último, no 
hay un momento ni un lugar para la participación - en cuyo caso resultaría algo anecdótico y de poco efecto educativo- sino que exige una continuidad y es factible de realizar en todas las fases, componentes y ámbitos de los procesos educativos (p. 32).

A través de una noción más centrada en el aula, Susinos y Ceballos (2012, p. 16) consideran que la participación son todas "aquellas iniciativas que emprenden las escuelas y que están dirigidas a aumentar el protagonismo del alumnado en la toma de decisiones sobre el diseño, la gestión y la evaluación de cualquier aspecto de la vida escolar".

De acuerdo con San Fabián (1994), existen múltiples maneras de comprender la participación del alumnado, las que van desde la colaboración hasta el consenso y la decisión conjunta entre estudiantes (Bretones, 1996, Fernández-Enguita, 1992).

Uno de los elementos fundamentales para analizar la participación es el grado de protagonismo de estudiantes en el aula. En la Tabla 1, se presenta una síntesis de Susinos y Ceballos (2012) sobre el grado de participación de estudiantes desde la mirada de distintos análisis.

Otro elemento importante del mismo tema son los ámbitos en los que pueden participar estudiantes. Rudduck y Flutter (2007) consideran que dicha participación puede centrarse tanto en la autonomía de los chicos y chicas, en la enseñanza de docentes o en elementos estrictamente curriculares (contenidos, objetivos, competencias, etc.) como también en los aspectos sociales e institucionales relacionados con la gestión de la escuela. Complementariamente, Smyth y Fasoli (2007) proponen una clasificación de los ámbitos de participación más amplios, como son la cultura o el clima escolar, la enseñanza, el aprendizaje o la estructura de la escuela. Por otra parte, Fielding y Bragg (2003) consideran que la participación estudiantil puede desarrollarse en un triple eje: en el proceso de enseñanza aprendizaje, es decir, en el aula, en la escuela y en la organización escolar. 
TABLA 1

Distintos niveles de protagonismo de estudiantes en las iniciativas de participación

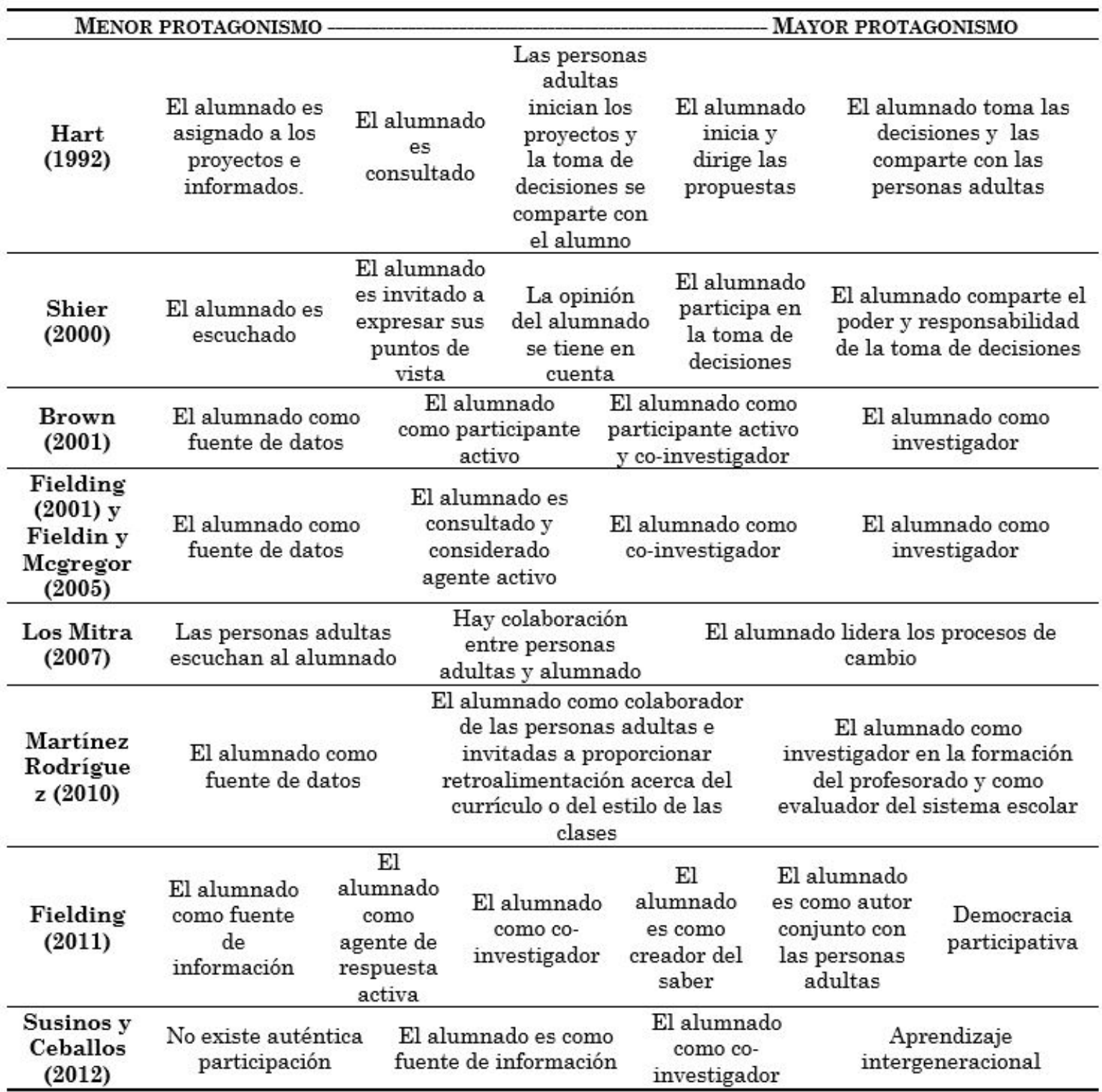

Nota: Recuperado de Susinos y Ceballos (2012, p. 28).

De acuerdo con lo señalado por Susinos y Ceballos (2012), la participación de estudiantes en las escuelas de España se relaciona "inevitablemente con la experiencia que han tenido los centros escolares en el funcionamiento de los consejos escolares y los representantes estudiantiles de aula o centro (delegados)" (p. 32). Asimismo, los autores añaden que dicha participación se suele implementar más en educación primaria y secundaria y en menor grado en educación infantil y en la enseñanza post-obligatoria (bachillerato y universidad).

Desde la perspectiva de Jagersma y Parsons (2011), la participación de estudiantes en su proceso de enseñanza tiene diversos beneficios para su aprendizaje y para su desarrollo integral. Algunos de ellos son los siguientes:

- Implicar a estudiantes con la planificación de las asignaturas y en la evaluación favorece su compromiso con su propio proceso de aprendizaje. 
- El aprendizaje estudiantil es constantemente presentado como elemento nuclear de la educación. Por lo tanto, si el objetivo es trabajar para mejorar el resultado de la enseñanza, el estudiantado debe ayudar a la producción del plan de estudios, y así sentirse más motivado e incluido (Levin, 2000).

- Si el estudiantado no se siente conectado con el plan de estudios o con los objetivos de un curso escolar, dicho plan de estudios se convertirá en una barrera para el aprendizaje (Flutter y Rudduck, 2004).

En la bibliografía revisada hemos encontrado numerosas investigaciones que profundizan en las prácticas de participación del profesorado, en relación con el proceso de enseñanza-aprendizaje de sus estudiantes. A continuación, presentamos de forma escueta el objetivo, metodología y principales resultados de los distintos estudios analizados.

La investigación internacional muestra los diversos beneficios que tiene la participación de estudiantes en la planificación y evaluación de su proceso de enseñanza. En esta línea, Sachs (2002) realiza un estudio cualitativo con 10 adolescentes en riesgo de exclusión social. En las entrevistas realizadas a este grupo encontró que la mayoría mencionó como uno de los elementos del fracaso escolar el poco grado de participación que sus docentes les permitían en el proceso de enseñanza. Esto generaba que no sintieran como propio aquello vivido en las aulas, sino como algo ajeno a su propio desarrollo.

En un trabajo más centrado en la labor del profesorado, Thompson (2009) lleva a cabo un estudio para determinar el papel docente en la participación de estudiantes en el aula, partiendo de la hipótesis de que el personal docente tiene un papel esencial en lograr la correcta implicación del estudiantado en el diseño de asignaturas y su evaluación. Así, el autor dividió a docentes participantes en el diseño en tres grupos, y en cada uno implementó un tipo de participación de estudiantes: en el primero una consulta proactiva (en la que se exigía una mayor participación), en el segundo una consulta de gestión y en el tercero una consulta más restringida (con muy poca participación). Los resultados mostraron que estudiantes del primer grupo valoraban emocionalmente el hecho de que sus docentes considerasen sus puntos de vista; al permitir su participación visibilizaron una conciencia mayor de su papel en el proceso de aprendizaje.

Un año más tarde Könings, Brand-Gruwel y Merrienboer (2010) llevan a cabo un estudio exploratorio para conocer los beneficios de la participación de estudiantes en el aula. La muestra estuvo conformada por seis docentes de dos escuelas secundarias de Países Bajos y sus estudiantes $(\mathrm{N}=139)$. Se realizaron grupos de trabajo en las seis aulas centrados en cómo debería ser la implicación de estudiantes en el diseño de las clases y se llevó a cabo la propuesta que acordaron. Los resultados evidenciaron que el estudiantado está absolutamente dispuesto y satisfecho de participar en el diseño del aprendizaje, y el profesorado afirmó que las propuestas del grupo de estudiantes eran muy interesantes. La principal conclusión de este estudio es que el diseño participativo es adecuado para su uso en educación, ya que favorece la autonomía, implicación y reflexión crítica del estudiantado.

Por medio de una investigación centrada en el uso de las propuestas de estudiantes en el aula como forma de participación, McIntyre, Pedder y Rudduck (2007) desarrollaron un estudio cualitativo para identificar cómo docentes usan las ideas y perspectivas que ofrecen sus estudiantes. El estudio se desarrolla en tres centros de educación secundaria y cuenta con la participación de dos docentes en cada uno. Los resultados mostraron que el estudiantado se centra más en su aprendizaje cuando siente que su docente implementa las ideas que propone o bien debate con el grupo para llegar a un consenso. En general, el profesorado utilizó las aportaciones de sus estudiantes al considerarlas muy útiles y prácticas. El personal docente que más usó las ideas de sus estudiantes notó que presentaban una mayor independencia y autonomía en su propio proceso de aprendizaje.

En el contexto español, Rojas, Haya y Lázaro-Visa (2012) realizaron un estudio cualitativo en dos centros educativos de Cantabria donde se trató de promover mejoras a partir de la participación de estudiantes en la enseñanza. Concretamente, se desarrollaron tres proyectos de investigación liderados por el alumnado de dos aulas de educación primaria, donde convirtieron al estudiantado en investigador y en partícipes de su 
propio proceso de aprendizaje. Se llevó a cabo una aproximación etnográfica a los centros para comprender en profundidad cómo se desarrollaba esta participación a través de entrevistas y sesiones de observación. Los resultados evidencian que el estudiantado se implicó y participó activamente en su proyecto mientras sus docentes les apoyaban. Los autores concluyen que el alumnado puede participar en asuntos del aula y de la escuela, con lo que se favorece su desarrollo y sentido de responsabilidad.

El análisis de los distintos estudios nos permite afirmar que la participación es un tema muy relevante a investigar por las implicaciones que tiene en el aprendizaje, implicación y motivación del alumnado. Desde nuestra perspectiva, la participación de estudiantes debería dar un paso más en las aulas españolas. Así, que el estudiantado participe no es únicamente escuchar sus distintos puntos de vista o consensuar un determinado aspecto de la enseñanza, sino implicarle activamente en el diseño de las actividades y la evaluación, participar en el desarrollo de las sesiones y tomar decisiones sobre su propio proceso de aprendizaje. En este sentido, abogamos por una participación orientada a la emancipación del alumnado y que le ayude a su desarrollo integral beneficioso, tanto para su vida académica como para su inserción social.

\section{Metodología}

\section{Objetivos de la investigación}

El presente estudio busca lograr los siguientes objetivos:

\section{Objetivo general}

Describir las prácticas pedagógicas y las actitudes que el personal docente tiene hacia la participación de sus estudiantes

Objetivos especificos:

1. Conocer las prácticas de participación que llevan a cabo docentes en el aula.

2. Identificar las actitudes que docentes tienen hacia la participación.

3. Conocer la relación entre las actitudes docentes y sus prácticas de participación.

4. Determinar si existen diferencias en las prácticas de participación de estudiantes en función de sus características sociodemográficas (género, situación laboral, área de conocimiento y comunidad autónoma).

\section{Enfoque metodológico}

Este estudio tiene un carácter cuantitativo y descriptivo, concretamente, se ha realizado un estudio ex post facto descriptivo. Este enfoque es el más adecuado para realizar esta investigación, ya que pretendemos tener una visión general de cómo estudiantes participan en las decisiones relativas tanto a la enseñanza como a la evaluación en el aula.

De acuerdo con Montero y León (2002), el uso de estudios ex post facto descriptivos/correlacionales es el más idóneo cuando el objetivo de la investigación es obtener una rica descripción sobre la participación del alumnado en su proceso de enseñanza-aprendizaje. Asimismo, posteriormente se la realizado un estudio relacional para conocer la relación entre las actitudes del profesorado hacia la participación y sus prácticas en el aula.

Complementando la visión de Montero y León (2002), Hernández, Fernández y Baptista (1998, p. 61) indican que un estudio descriptivo "mide de manera más bien independiente los conceptos o variables a los que se refiere. Su objetivo no es indicar cómo se relacionan las variables medidas". Además, los propios autores indican que "la investigación correlacional tiene, en alguna medida, un valor explicativo aunque parcial. Saber 
que dos conceptos o variables están relacionados aporta cierta información explicativa.” (p. 65), y abarca los tres objetivos propuestos, ya que también se va a medir la relación entre las actitudes docentes y las prácticas de participación del alumnado incentivadas por sus docentes.

Las variables, surgidas a partir de la revisión teórica acerca de cuáles son las principales prácticas de participación de estudiantes en el aula, son:

- Características personales y profesionales del personal docente.

- Características de los centros educativos.

- Actitudes de docentes hacia la participación.

- Prácticas de docentes relacionadas con la participación de estudiantes en:

- La selección del contenido a enseñar

- Las estrategias didácticas a llevar a cabo

- La evaluación de los aprendizajes

\section{Población, muestra y muestreo}

La muestra del presente estudio es de 177 docentes de educación secundaria que imparten clases en 10 institutos de educación secundaria (IES) situados en las comunidades de Andalucía, Castilla y León, Extremadura y Madrid. La selección de estas comunidades responde a que son regiones con diferentes características sociales, culturales y económicas. En este sentido, la muestra utilizada es una representación significativa de la diversidad de docentes de educación secundaria de España. Se eligieron cuatro comunidades autónomas con unas características socioeconómicas diferentes que representan las distintas comunidades del país.

El muestreo ha sido estratificado por conglomerados a partir de comunidades autónomas y niveles. Así, las escuelas se eligieron de forma estratificada constante y el conglomerado fue el aula del centro educativo, la unidad a la que se le proporcionó el cuestionario.

En cuanto a las características de la muestra, se analizó: el género de docentes, la comunidad autónoma de la que proceden, los años de experiencia, el área donde dan clases, la formación previa y su situación laboral en el centro educativo en el momento de realizar la investigación. En cuanto al género, el 41,1\% de la muestra son hombres frente al 58,9\% de mujeres. La media de experiencia docente es de 20,5 años, una parte del profesorado se encontraba en su primer año de enseñanza y otra llevaba hasta 45 años de práctica profesional. En la Tabla 2 describimos la distribución de docentes en función de la comunidad autónoma, donde Castilla y León son las comunidades con un mayor porcentaje de profesores y profesoras, y el País Vasco con el menor número.

TABLA 2

Tabla de distribución de docentes en función de la comunidad autónoma

\begin{tabular}{lcc}
\hline \multicolumn{1}{c}{ ComUNIDAD AUTÓNOMA } & Docentes & PoRCENTAJE \\
\hline Andalucía & 31 & $17,5 \%$ \\
Castilla y León & 46 & $26 \%$ \\
Extremadura & 43 & $24,3 \%$ \\
Madrid & 38 & $21,5 \%$ \\
País Vasco & 19 & $10,7 \%$ \\
Total & 177 & $100 \%$ \\
\hline
\end{tabular}


Si se analiza la situación laboral del profesorado participante, vemos que el 64,2\% es titular con destino definitivo en el centro educativo, un $20,2 \%$ es interino, un $10,4 \%$ titulares con destino provisional en ese instituto y un 5,2\% está en comisión de servicios. Los datos que reflejan la formación previa que ha recibido el personal docente nos muestran que la mayoría cuenta con licenciatura $(88,7 \%)$, varios con diplomado $(17,1 \%)$ y un pequeño porcentaje con doctorado $(5,1 \%)$.

La última variable sociodemográfica analizada de la muestra es el departamento en el que este grupo de docentes imparte la docencia. Los estadísticos descriptivos nos permiten afirmar que el área con más profesorado es matemáticas, seguida por lengua y ciencias sociales. En contraposición, música y euskera son los departamentos con menor presencia en nuestro estudio (Figura 1).

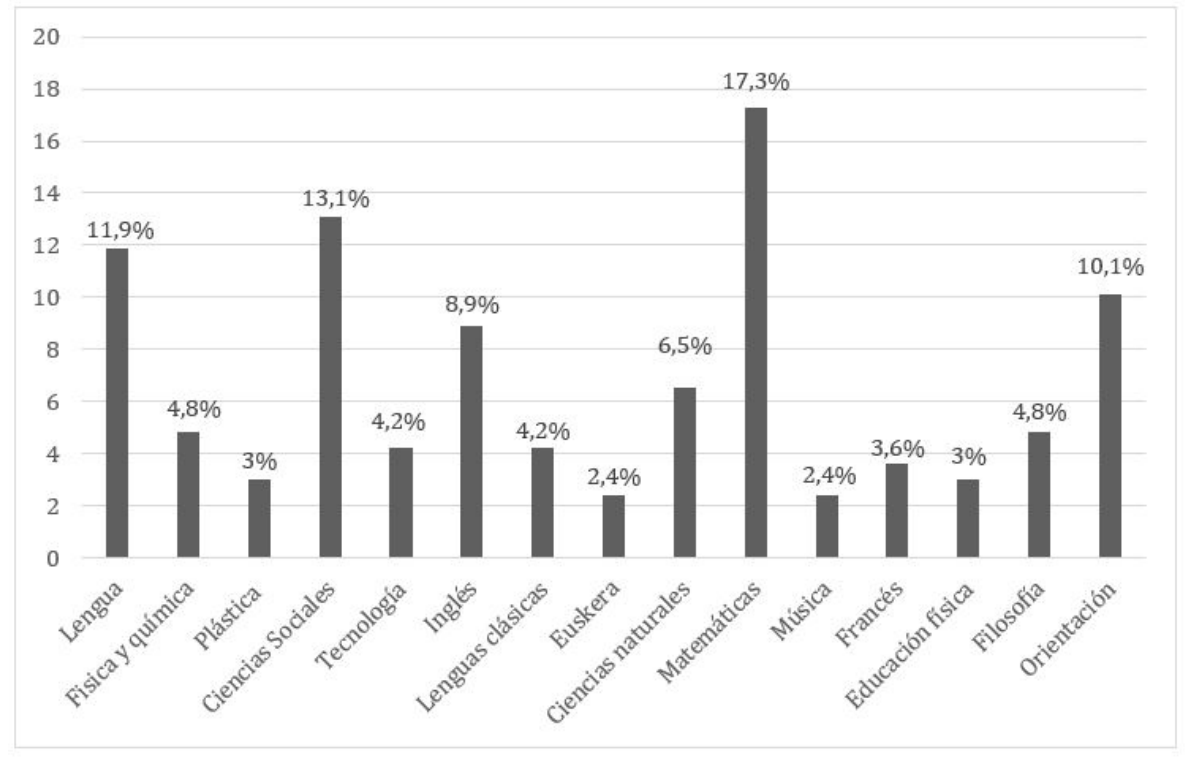

FIGURA 1

Distribución de docentes en función del departamento en el que imparten su enseñanza. Elaboración propia.

\section{Instrumento, recogida de la información y análisis de datos}

El instrumento utilizado en la presente investigación es una parte de un cuestionario que busca conocer las prácticas de enseñanza y evaluación de docentes de educación secundaria. El cuestionario contiene diversos ítems referidos a la participación de estudiantes en la enseñanza y en la evaluación. Asimismo, el cuestionario contiene una escala de actitudes de docentes hacia la justicia social, donde también se incluyen varias preguntas relacionadas con dar voz al alumnado. El cuestionario completo está compuesto por 23 ítems y en este estudio nos centramos en analizar 12 de ellos.

Concretamente, para la consecución de los objetivos que nos hemos propuesto se tuvieron en consideración las siguientes variables:

- Datos sociodemográficos de cada docente (comunidad autónoma, género, años de experiencia, situación laboral, formación previa y área de conocimiento)

- Actitudes de docentes hacia la participación y sus prácticas.

- Prácticas de participación de estudiantes llevadas a cabo por docentes en el aula.

Las preguntas en las que nos centramos son de tres tipos: a) algunas dicotómicas, con dos alternativas de respuesta, b) otras politómicas, con varias alternativas nominales y c) tipo Likert, con cinco niveles de alternativa de respuesta. 
Antes de su aplicación, el cuestionario fue validado en dos fases (validez de contenido y de constructo). La primera fase supuso una aplicación piloto del cuestionario con un profesor, quien nos expresó aquellas preguntas que debían sufrir cierto replanteamiento o modificación. Una vez corregida esta primera versión del cuestionario, se realizó una aplicación piloto en la segunda fase. Para ello, se aplicó el cuestionario a un aula de 25 docentes de educación secundaria a quienes posteriormente se les dio un espacio para comentar los aspectos en los que habían tenido alguna dificultad para responder. Estos resultados preliminares y los comentarios realizados nos ayudaron a diseñar la versión final del cuestionario más ajustada a la realidad del alumnado.

En términos psicométricos, la escala de actitudes (que conforma una parte del cuestionario) tiene una fiabilidad alta de un Alpha de Cronbach de 0,83. Esta fiabilidad se ha obtenido mediante un análisis estadístico de todas las preguntas del cuestionario de forma conjunta, de lo que se obtuvo un índice de fiabilidad del instrumento utilizado. De acuerdo con Loo (2001) o Gliem y Gliem (2003), el instrumento se considera fiable a partir de 0,7 , por lo tanto, resultó adecuado para la realización de este estudio.

Para el análisis de los datos hemos utilizado análisis de tipo descriptivos e inferenciales a través del paquete estadístico SPSS versión 22. Los análisis utilizados para este estudio han sido:

a) Estadística descriptiva: descripción y frecuencias de los resultados de las actitudes docentes hacia la participación y el grado de participación del alumnado en la enseñanza y la evaluación,

b) Estadistica inferencial: T-Student para determinar las diferencias por género en las actitudes y el grado de participación, y Anova de un factor para conocer las diferencias en cuanto al área de conocimiento, la situación laboral y la comunidad autónoma de procedencia del personal docente.

c) Estadistica correlacional: correlación para identificar la relación entre la actitud de docentes hacia la participación y sus prácticas concretas para incluir a sus estudiantes en la enseñanza y la evaluación.

\section{Resultados}

La presentación de los resultados se organiza a partir de los siguientes temas:

a) Actitudes de docentes hacia la participación de estudiantes

b) Participación de estudiantes en la enseñanza y en la evaluación

c) Relación entre las actitudes de docentes y sus prácticas de participación, y relación entre las distintas prácticas de participación (enseñanza, selección de contenido y participación).

En los resultados mostrados, también se mide si existen diferencias significativas en las prácticas de enseñanza y participación que llevan a cabo docentes en función del género, área de conocimiento, situación laboral y comunidad autónoma.

En los dos primeros apartados, analizamos en profundidad si existen diferencias en función de las características sociodemográficas.

\section{Actitudes de los docentes hacia la participación de los estudiantes}

La gran mayoría del profesorado es enfática al defender que una de sus prioridades es fomentar la participación estudiantil. Así, un 93,0\% de docentes de educación secundaria está de acuerdo con esta afirmación (Figura 2). 


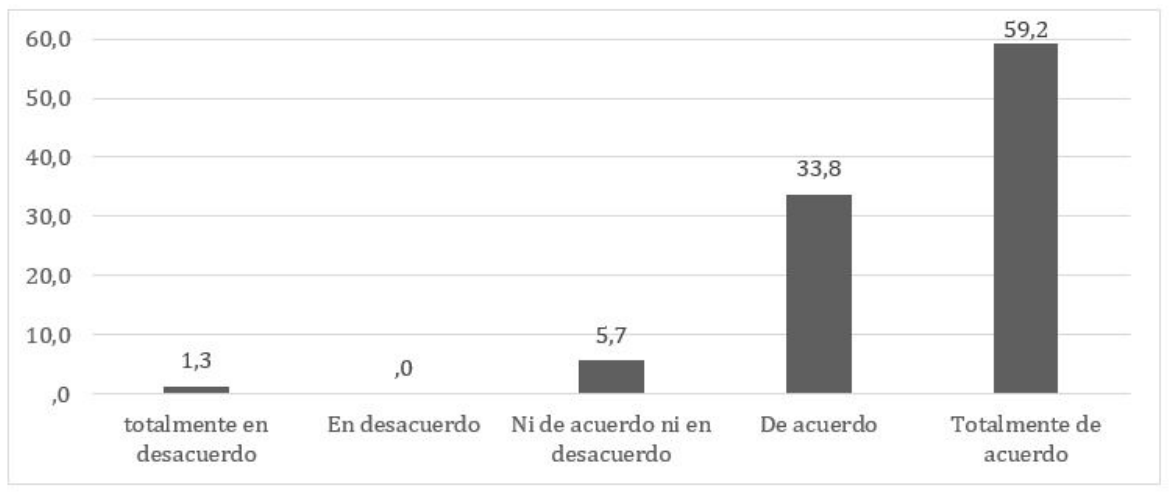

FIGURA 2

Distribución de docentes en su actitud hacia la participación de sus estudiantes Elaboración propia

Si profundizamos en cómo se comporta esta variable en relación con el género de la muestra, podemos afirmar que son las profesoras quienes tienen una actitud más positiva hacia la participación en comparación con los hombres $($ sig. $=<0,05)$, con una media d 4,61 frente a 4,37 (Tabla 3 ).

TABLA 3

Resultados de la aplicación de la prueba T-Student. Diferencias de género en la actitud de docentes hacia la participación

\begin{tabular}{l|c|c|c|c}
\hline & Hombre & Mujer & T & Sig. \\
\hline & & & & \\
& & 4,37 & $-2,264$ & 0,025 \\
$\begin{array}{l}\text { 11. Una de mis prioridades como } \\
\text { docente es fomentar la participación } \\
\text { de mis estudiantes }\end{array}$ & & & \\
\hline
\end{tabular}

Nota: $\left(^{*}\right)$ Asumimos varianzas iguales, prueba de Levene $(\operatorname{sig}=0,05)$. Elaboración propia.

El análisis de las diferencias en función de la situación laboral nos permite afirmar que existen diferencias entre personal interino, titular definitivo y provisional y docente en comisión de servicios (Tabla 4). Los análisis post-hoc realizados a posteriores con el estadístico Tukey confirman que el grupo de docentes titulares con destino provisional son quienes tienen una actitud más favorable hacia la participación estudiantil $(\mathrm{m}=4,78)$, seguido por el de profesorado interino $(\mathrm{m}=4,68)$. El personal docente en comisión de servicios es el que muestra una actitud más baja de $(\mathrm{m}=4,11)$ en relación al mismo tema.

\section{TABLA 4}

Resultados de la aplicación de la prueba Anova de un factor. Diferencias en función de la situación laboral en la actitud de docentes hacia la participación

\begin{tabular}{l|l|l|l|l}
\hline & $\begin{array}{l}\text { Inter- } \\
\text { grupos }\end{array}$ & $\begin{array}{l}\text { Intra- } \\
\text { grupos }\end{array}$ & F & Sig. \\
\hline & & & & \\
$\begin{array}{l}\text { Una de mis prioridades como } \\
\text { docente es fomentar la participación } \\
\text { de mis estudiantes }\end{array}$ & & & & \\
\hline
\end{tabular}


Para determinar si existen diferencias en función del departamento en el que imparten docencia, hemos agrupado las asignaturas en áreas de conocimiento. Los resultados muestran que no existen diferencias en función del departamento en cuanto a la participación de estudiantes en el aula (Tabla 5).

TABLA 5

Resultados de la aplicación de la prueba Anova de un factor. Diferencias en función del área de conocimiento en la actitud de docentes hacia la participación

\begin{tabular}{l|l|l|l|l}
\hline & $\begin{array}{l}\text { Inter- } \\
\text { grupos }\end{array}$ & $\begin{array}{l}\text { Intra- } \\
\text { grupos }\end{array}$ & F & \\
\hline & & & Sig. \\
\hline $\begin{array}{l}\text { 11. Una de mis prioridades como } \\
\text { docente es fomentar la participación } \\
\text { de mis estudiantes }\end{array}$ & 1,82 & 83,93 & 1,18 & \\
\hline
\end{tabular}

Nota: Elaboración propia.

$\mathrm{Al}$ analizar si hay diferencias en la actitud hacia la participación de docentes y los años de experiencia, podemos afirmar que estas no existen (sig.=0,36). Por último, respecto a si existen diferencias por la comunidad autónoma donde imparten la docencia, los resultados muestran que tampoco existen tales diferencias, dado que la significación es superior a 0,05 (Tabla 6).

TABLA 6

Resultados de la aplicación de la prueba Anova de un factor. Diferencias en función de la comunidad autónoma en la actitud de docentes hacia la participación

\begin{tabular}{l|l|l|l|l}
\hline & $\begin{array}{l}\text { Inter- } \\
\text { grupos }\end{array}$ & $\begin{array}{l}\text { Intra- } \\
\text { grupos }\end{array}$ & F & Sig. \\
\hline & & & & \\
& & & & \\
$\begin{array}{l}\text { 11. Una de mis prioridades como } \\
\text { docente es fomentar la participación } \\
\text { de mis estudiantes }\end{array}$ & 0,89 & 87,10 & 0,44 & 0,78 \\
\hline
\end{tabular}

Nota: Elaboración propia.

\section{Participación de estudiantes en la enseñanza y en la evaluación}

Tal como hemos señalado, las acciones que los grupos docentes realizan en relación con la participación se han organizado en tres elementos, a) la selección de los contenidos a enseñar, a) el diseño de las estrategias didácticas de las diferentes materias; b) el diseño de las estrategias de evaluación. 


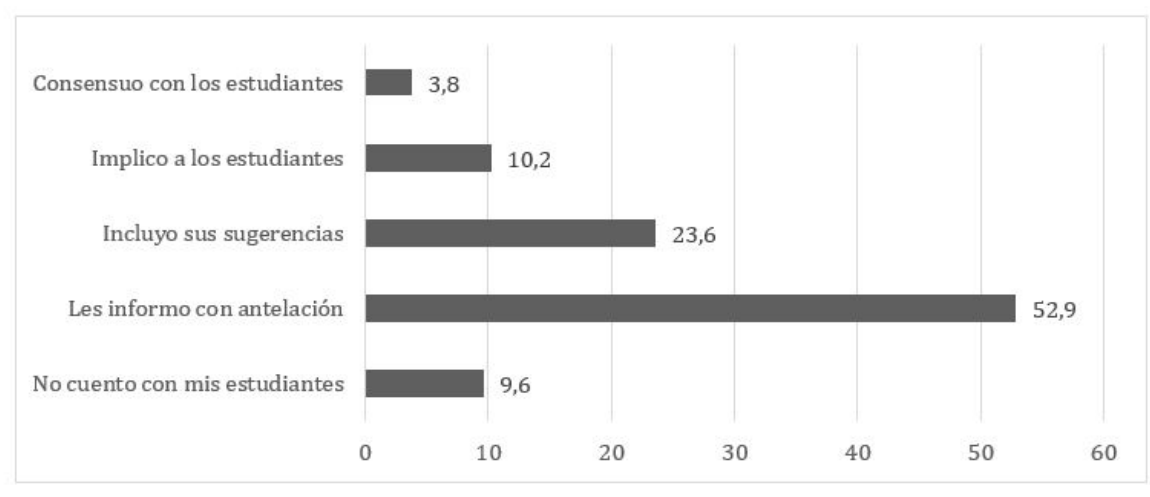

FIGURA 3

Participación de estudiantes en la selección de contenidos a enseñar. Nota: Elaboración propia.

Los datos muestran que la participación de estudiantes en los contenidos a enseñar es el ámbito donde el profesorado les permiten participar en menor medida. Así, tal como muestran la Figura 3 más de la mitad de docentes $(52,9 \%)$ se limita a informar a sus estudiantes de los contenidos a trabajar en el aula, seguido de un 23,6\% de docentes que incluye sugerencias del alumnado en los contenidos a desarrollar en el aula. Por otro lado, implicar o consensuar con estudiantes los contenidos a enseñar es llevado a cabo solamente por un $10,2 \%$ y un $3,8 \%$ respectivamente.

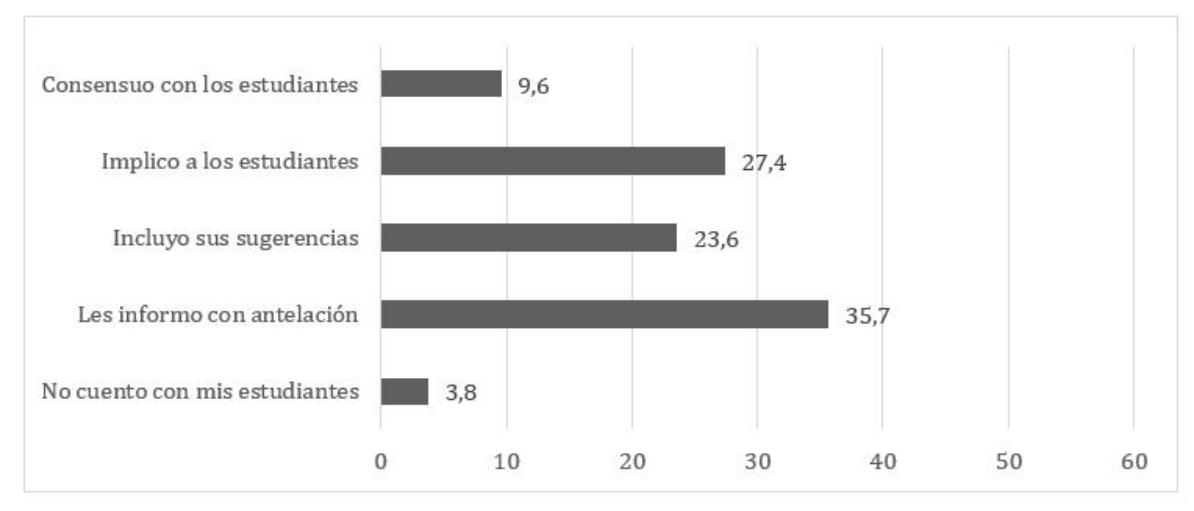

FIGURA 4

Participación de estudiantes en el diseño de estrategias didácticas

Nota: Elaboración propia

En el Figura 4 se observan las prácticas que realizan los grupos docentes para que sus estudiantes participen en el diseño de las estrategias de enseñanza que se llevan a cabo en el aula. Las alternativas que se ofrecen a docentes en el cuestionario, incluyen cinco posibilidades de implicación de estudiantes; desde una nula participación hasta un absoluto consenso de las acciones a realizar en el aula. Los resultados muestran que un $35,7 \%$ de docentes se limita a informar a sus estudiantes sobre la estrategia didáctica a seguir, sin tener en cuenta su opinión ni tampoco dándoles la oportunidad de participar.

Quienes sí permiten la participación en el aula, son un $51 \%$ de la muestra. Dentro de esta participación, un $23,6 \%$ de docentes incluye las sugerencias de estudiantes en el diseño de las clases y un 27,4\% les implica en el diseño de las estrategias a llevar a cabo en el aula.

Solamente un $9,6 \%$ del profesorado dialoga con sus estudiantes la totalidad las estrategias didácticas a desarrollar en el aula. 




FIGURA 5

Participación de estudiantes en el diseño de estrategias de evaluación

Nota: Elaboración propia

En cuanto a las acciones que llevan a cabo docentes para promover la participación de estudiantes en las estrategias de evaluación, observamos en el Figura 5 resultados diferentes a la implicación del alumnado en las estrategias didácticas mencionadas en el apartado anterior.

Más de la mitad de docentes (58\%) informa a sus estudiantes de las estrategias de evaluación que va a realizar, sin permitir su participación en la forma en cómo va a evaluarles. Un $18,5 \%$ de docentes incluye las sugerencias de sus estudiantes en las estrategias de evaluación, un 11,5\% implica a estudiantes y un $7 \%$ consensua con el grupo las estrategias de evaluación a utilizar respectivamente. Si comparamos la participación de estudiantes en las tres prácticas analizadas (selección del contenido, diseño de las estrategias didácticas y diseño de la evaluación) podemos observar que el personal docente permite que sus estudiantes participen, en mayor medida, en la selección de las estrategias didácticas y, en menor medida, en la evaluación o en la selección de los contenidos. Así, un 35,8\% consensua o implica al alumnado en la enseñanza, frente a un $17,6 \%$ en la evaluación y un $14,2 \%$ en los contenidos. En coherencia con estos datos, los contenidos también son el ámbito donde los profesores y profesoras informan en menor medida a sus estudiantes (9,1\%), seguida por la evaluación $(4,5 \%)$ y la enseñanza $(3,8 \%)$.

En las prácticas de participación analizadas (enseñanza, selección del contenido y evaluación), la opción más elegida por los profesores y profesoras es informar al alumnado con antelación de lo que se va a llevar a cabo.

Analizando si existen diferencias en la participación en las tres prácticas docentes analizadas, podemos afirmar que en cuanto al género no existen diferencias (Tabla 7). Lo mismo sucede en los años de experiencia docente, pudiendo observar que no existe ninguna significación inferior a 0,05 en la selección de contenidos, estrategias de enseñanza y de evaluación (con una sig. de 0,07, 0,025 y 0,053 respectivamente).

TABLA 7

Resultados de la aplicación de la prueba T-Student. Diferencias de género en las prácticas de participación de estudiantes

\begin{tabular}{l|c|c|c|c}
\hline & Hombre & Mujer & T & Sig. \\
\hline $\begin{array}{l}\text { Participación estudiantes - estrategias } \\
\text { didácticas }\end{array}$ & 2,94 & 3,1 & $-0,94$ & 0,35 \\
\hline $\begin{array}{l}\text { Participación estudiantes - estrategias de } \\
\text { evaluación }\end{array}$ & 2,43 & 2,66 & $-1,49$ & 0,14 \\
\hline $\begin{array}{l}\text { Participación estudiantes - contenidos a } \\
\text { enseñar }\end{array}$ & 2,43 & 2,47 & $-0,28$ & 0,78 \\
\hline
\end{tabular}


En cuanto a las diferencias en la participación de estudiantes en la selección de los contenidos, la enseñanza, la evaluación y el área de conocimiento donde imparten docencia y su situación laboral vemos que, respecto a la primera de las características, no existen diferencia de medias en relación con la materia que imparten (Tabla 8). Tampoco hemos hallado diferencias en su situación laboral, por lo que los profesores y profesoras consideran la participación del alumnado independientemente de su relación contractual con el centro educativo (Tabla 9). Por último, podemos afirmar que la comunidad autónoma tampoco es un factor que determine diferencias en la forma de incluir a estudiantes en el aula (Tabla 10).

TABLA 8

Resultados de la aplicación de la prueba Anova de un factor. Diferencias en función del área de conocimiento en la actitud de docentes hacia la participación estudiantil

\begin{tabular}{l|c|c|c|c}
\hline & $\begin{array}{c}\text { Inter- } \\
\text { grupos }\end{array}$ & $\begin{array}{c}\text { Intra- } \\
\text { grupos }\end{array}$ & F & Sig. \\
\hline $\begin{array}{l}\text { Participación de estudiantes en el } \\
\text { diseño de las estrategias didácticas }\end{array}$ & 7,73 & 181,12 & 2,32 & 0,77 \\
\hline $\begin{array}{l}\text { Participación de estudiantes en el } \\
\text { diseño de las estrategias de } \\
\text { evaluación }\end{array}$ & 6,62 & 153,69 & 1,98 & 0,12 \\
\hline $\begin{array}{l}\text { Participación de estudiantes en la } \\
\text { selección de contenidos }\end{array}$ & 1,9 & 143,06 & 0,72 & 0,54 \\
\hline
\end{tabular}

Nota: Elaboración propia

TABLA 9

Resultados de la aplicación de la prueba Anova de un factor. Diferencias en función de la situación laboral en actitud de docentes hacia la participación estudiantil

\begin{tabular}{|c|c|c|c|c|}
\hline & $\begin{array}{l}\text { Inter- } \\
\text { grupos }\end{array}$ & $\begin{array}{l}\text { Intra- } \\
\text { grupos }\end{array}$ & F & Sig. \\
\hline $\begin{array}{l}\text { Participación de estudiantes en el } \\
\text { diseño de las estrategias didácticas }\end{array}$ & 5,4 & 186,50 & 1,62 & 0,19 \\
\hline $\begin{array}{l}\text { Participación de estudiantes en el } \\
\text { diseño de las estrategias de } \\
\text { evaluación }\end{array}$ & 0,18 & 162,34 & 0,64 & 0,98 \\
\hline $\begin{array}{l}\text { Participación de estudiantes en la } \\
\text { selección de contenidos }\end{array}$ & 2,71 & 145,82 & 1,04 & 0,38 \\
\hline
\end{tabular}


TABLA 10

Resultados de la aplicación de la prueba Anova de un factor. Diferencias en función de las comunidades autónomas en actitud de docentes hacia la participación estudiantil

\begin{tabular}{l|c|c|c|c}
\hline & $\begin{array}{c}\text { Inter- } \\
\text { grupos }\end{array}$ & $\begin{array}{c}\text { Intra- } \\
\text { grupos }\end{array}$ & F & Sig. \\
\hline $\begin{array}{l}\text { Participación de estudiantes en el } \\
\text { diseño de las estrategias didácticas }\end{array}$ & 3,35 & 194,56 & 0,74 & 0,57 \\
\hline $\begin{array}{l}\text { Participación de estudiantes en el } \\
\text { diseño de las estrategias de } \\
\text { evaluación }\end{array}$ & 2,48 & 166,95 & 0,63 & 0,64 \\
\hline $\begin{array}{l}\text { Participación de estudiantes en la } \\
\text { selección de contenidos }\end{array}$ & 2,52 & 155,28 & 0,69 & 0,6 \\
\hline
\end{tabular}

Nota: Elaboración propia

\section{Relación entre las actitudes y la participación de estudiantes en el aula}

Una vez que hemos estudiado las actitudes hacia la participación y las prácticas concretas de enseñanza, evaluación y selección de contenido queremos conocer si existe relación entre ambas. En ese sentido, se ha analizado si existe relación entre la actitud de docentes hacia la participación y su práctica docente. Asimismo, también se ha analizado si existe relación entre las prácticas de participación analizadas (selección de los contenidos, la enseñanza, la evaluación) (Tabla 11).

$\mathrm{Al}$ respecto, podemos observar que la forma en que el personal docente permite la participación de sus estudiantes en el diseño de las estrategias didácticas correlaciona positivamente con el diseño de las estrategias de evaluación $(\mathrm{r}=0,512)$ y la selección de contenidos a enseñar $(\mathrm{r}=0,431)$. Los contenidos a enseñar también correlacionan positivamente con el diseño de las estrategias de evaluación $(r=0,479)$.

TABLA 11

Correlación entre las prácticas diferentes de participación

\begin{tabular}{l|c|c|c|c}
\hline & & $\begin{array}{c}\text { Diseño de } \\
\text { estrategias } \\
\text { didácticas }\end{array}$ & $\begin{array}{c}\text { Diseño de } \\
\text { estrategias } \\
\text { de } \\
\text { evaluación }\end{array}$ & $\begin{array}{c}\text { Contenidos } \\
\text { a enseñar }\end{array}$ \\
\hline $\begin{array}{c}\text { Diseño de } \\
\text { estrategias } \\
\text { didácticas }\end{array}$ & Correlación de Pearson & 1 & $0,512 * *$ & $0,431^{* *}$ \\
\cline { 2 - 5 } & Sig. (bilateral) & & 0,00 & 0,00 \\
\hline $\begin{array}{l}\text { Diseño de } \\
\text { estrategias de } \\
\text { evaluación }\end{array}$ & Correlación de Pearson & $0,512^{* *}$ & 1 & $0,479 * *$ \\
\cline { 2 - 5 } $\begin{array}{c}\text { Contenidos a } \\
\text { enseñar }\end{array}$ & Sig. (bilateral) & 0,00 & & 0,00 \\
\cline { 2 - 5 } & Correlación de Pearson & $0,431 * *$ & $0,479 * *$ & 1 \\
\hline
\end{tabular}

Nota: ${ }^{* *}$ La correlación es significativa al nivel 0,01 (bilateral). Elaboración propia.

A su vez, hemos analizado la correlación existente en las actitudes docentes y sus acciones (Tabla 12); es decir, su posición frente a la afirmación Una de mis prioridades como docente es fomentar la participación de estudiantes y sus acciones docentes en cuanto a participación real de estudiantes en el aula. Los datos muestran que la correlación más fuerte es la existente entre su actitud y los contenidos a enseñar $(\mathrm{r}=$ 0,270), aún sin ser una correlación muy alta. En el diseño de las estrategias de enseñanza y de evaluación 
no encontramos una correlación significativa con su actitud frente a la participación. Estos resultados nos permiten confirmar la distancia que existe entre las actitudes de docentes y la propia práctica.

TABLA 12

Relación entre las actitudes y las prácticas de participación

\begin{tabular}{l|c|c}
\hline & \multicolumn{2}{|c}{$\begin{array}{c}\text { Una de mis prioridades como docente es } \\
\text { fomentar la participación de mis } \\
\text { estudiantes }\end{array}$} \\
\hline & Correlación de Pearson & Sig. (bilateral) \\
\hline Diseño de estrategias didácticas & 0,148 & 0,050 \\
\hline Diseño de estrategias de evaluación & 0,113 & 0,138 \\
\hline Contenidos a enseñar & $0,270 * *$ & 0,000 \\
\hline
\end{tabular}

Nota: ${ }^{* *}$ La correlación es significativa al nivel 0,01 (bilateral). Elaboración propia.

\section{Discusión}

Los hallazgos de nuestra investigación apuntan a que la participación estudiantil sigue siendo una materia pendiente dentro de las prácticas del profesorado. Ha quedado en evidencia que la implicación del alumnado no es suficiente y que faltan acciones más intencionadas por la parte docente para lograr que sus estudiantes no solo opinen sobre algunas estrategias didácticas, sino que también tengan voz en los contenidos y en la evaluación.

En términos generales, los hallazgos de este estudio confirman las aportaciones de trabajos anteriores respecto a la escasa implicación de estudiantes en su proceso de educativo (Sachs, 2002; Susinos y Ceballos, 2012; Thompson, 2009). Por ejemplo, Susinos y Ceballos (2012) consideran que, en España, la participación de estudiantes se observa en mayor medida en la educación primaria a través de elementos como la integración en el consejo escolar o en la asamblea, lo que deja en un segundo plano la participación en el diseño del proceso de enseñanza-aprendizaje.

Asimismo, la poca relación que existe entre las actitudes y las acciones de docentes que ha quedado reflejada en este estudio, es coherente con lo que afirmaba Fernández Enguita (1992). Para este autor la participación se encuentra más en el discurso del profesorado que en sus prácticas reales. En otras palabras, una cosa es lo que dicen y otra muy distinta es lo que hacen.

También vemos algunas diferencias entre los resultados de esta investigación y los de estudios anteriores. En el caso de McIntyre, Pedder y Rudduck (2007), los hallazgos son más optimistas que los del presente trabajo, ya que el profesorado utilizón las aportaciones de sus estudiantes al considerarlas muy útiles y prácticas. Lo mismo sucede con la investigación de los españoles Rojas, Haya y Lázaro-Visa (2012), donde el estudiantado se implicó y participó activamente en su proyecto mientras sus docentes le apoyaban.

\section{Conclusiones}

Los resultados del presente estudio aportan evidencias empíricas de que existe una enorme distancia entre las actitudes de docentes hacia la participación de sus estudiantes y sus prácticas reales en el aula. Llama la atención que la mayoría del profesorado afirma estar totalmente de acuerdo en que una de sus prioridades es favorecer la participación de sus estudiantes en el aula. No obstante, cuando se les pregunta cómo les incluyen en la selección de contenidos, la elección de estrategias didácticas o en los instrumentos de evaluación, la respuesta es rotundamente contraria: la mayoría de docentes solamente informa con antelación al alumnado lo relativo a estos temas o en algún caso incluyen sus sugerencias. Así, podemos afirmar que existe una 
diferencia notable entre lo que los grupos docentes piensan y creen sobre la participación y cómo desarrollan este concepto en la realidad del aula.

Esta investigación es una pequeña aportación acerca de las actitudes hacia la participación y las prácticas de participación de ldocentes en el contexto español y desde ese punto de vista nos parece relevante continuar ampliando este tema. Para continuar profundizando en los hallazgos de este trabajo sería interesante realizar un estudio cualitativo de enfoque fenomenográfico centrado en las creencias de participación del personal docente, o en los motivos por los que cree que la participación no se implementa de forma real en las aulas. Otra investigación que arrojaría datos relevantes sería un estudio tanto ex post facto como cualitativo con estudiantes, para comprender sus creencias y experiencias de participación en la educación y los beneficios de aprendizaje que reportan desde su perspectiva.

La participación estudiantil debe convertirse en un tema prioritario para el profesorado y para la educación en su conjunto. Nuestro alumnado no puede ser observado como un mero receptor inmóvil de nuestras decisiones; por el contrario, deben sentirse implicado en su formación. No basta con informarle los contenidos, la normativa, o los criterios de evaluación; su implicación real tiene que ver con procesos de diálogo, debate y consenso en un ambiente de entendimiento mutuo. Las relaciones asimétricas entre docentes y estudiantes se deben adaptar paulatinamente hacia formas de comunicación menos jerárquicas y más flexibles. No es tan ilusorio pensar que las palabras de Fernández Enguita (1992) mencionadas en la introducción de este trabajo puedan cambiar en un futuro próximo, y lograr que la expresión "participación en educación" deje de ser un término ilusorio y se convierta en una realidad concreta y tangible en nuestras aulas.

\section{ReFERENCIAS}

Allwright, D. (1984). Why don't learners learn what teachers teach? The interaction hypothesis. Language Learning in Formal and Informal Contexts, 1, 3-18.

Bernstein, B. (1990). Class, codes and control, vol. 4: The structuring of pedagogic. Londres: Routledge.

Bretones, A. (1996). Concepciones y prácticas de participación en el aula según los estudiantes de magisterio (Tesis doctoral). Universidad Complutense de Madrid, Madrid, España.

Cook-Sather, A. (2001). Unrolling roles in techno-pedagogy: Toward new forms of collaboration in traditional college settings. Innovative Higher Education, 26, 121-39.

Cook-Sather, A. (2002). Authorizing students' perspectives: Toward trust, dialogue, and change in education. Educational Researcher, 31(4), 3-14.

Fernández Enguita, M. (1992). Poder y participación en el sistema educativo. Madrid: Paidós.

Fielding, M. y Bragg, S. (2003). Students as researchers: Making a difference. Cambridge: Pearson Publishing.

Flutter, J. y Rudduck, J. (2004). Consulting Pupils: What's in it for Schools? Londres: Psychology Press.

Fraser, N. (2008). La justicia social en la era de la política de identidad: Redistribución, reconocimiento y participación. Revista de Trabajo, 4(6), 83-99.

Fredricks, J. A., Blumenfeld, P. C., y Paris, A. H. (2004). School engagement: Potential of the concept, state of the evidence. Review of Educational Research, 74(1), 59-109.

Gliem, J. A. y Gliem, R. R. (2003). Calculating, interpreting, and reporting Cronbach's alpha reliability coefficient for Likert-type scales. Comunicación presentada en el Midwest Research- to-Practice Conference in Adult, Continuing, and Community Education, Columbus, $\mathrm{OH}$.

Hernández, . R., Fernández, F. y Baptista, P. (1998). Metodología de la investigación. Ciudad de México: Mc GrawHill Editores.

Jagersma, J. y Parsons, J. (2011). Empowering Students as Active Participants in Curriculum. Design and Implementation. New Zealand Journal of Teachers' Work, 8(2), 114 -121. 
Könings, K.D., Brand-Gruwel, S., y Merriënboer, J.J. (2010). An approach to participatory instructional design in secondary education: an exploratory study. Educational Research, 52(1), 45-59.

Leithwood, K., Mulford, W. y Silins, H. (2004). Educational leadership for organisational learning and improved student outcomes. Londres: Kluwer Academic Publishers. Dordrecht.

Levin, B. (2000). Putting students at the centre in education reform. Journal of Educational Change, 1(2), 155-172.

Loo, R. (2001). Motivational Orientations toward Work: An Evaluation of the Work Preference Inventory (Student Form). Measurement and Evaluation in Counseling and Development, 33, 222-233.

McIntyre, D., Pedder, D. y Rudduck, J. (2007). Pupil voice: comfortable and uncomfortable learnings for teachers. Research Papers in Education, 20(2), 149-168.

Mitra, DL. y Gross, S.J. (2009). Increasing student voice in high school reform: Building partnerships, improving outcomes. Educational Management Administration \& Leadership, 37 (4), 522-543.

Montero, I. y León, O. G. (2002). Clasificación y descripción de las metodologías de investigación en psicología. International journal of clinical and health psychology, 2(3), 503-508.

Morell, T. (2004). Assessment and online feedback:: using a written text correction programme. Porta Linguarum: Revista Internacional de Didáctica de las Lenguas Extranjeras, 3, 123-134.

Morell, T. (2007). La integración de los alumnos en el proceso de evaluación. Actas del XXIV Congreso Internacional de AESLA: Aprendizaje de lenguas, uso del lenguaje y modelación cognitiva: perspectivas aplicadas entre disciplinas.

Morell, T. (2009). ¿Cómo podemos fomentar la participación en nuestras clases universitarias?. Madrid: Marfil.

Murillo, F.J. y Hernández-Castilla, R. (2011). Hacia un concepto de justicia social. REICE. Revista Iberoamericana sobre Calidad, Eficacia y Cambio en Educación, 9(4), 7-23.

Murillo, F.J. y Hernández-Castilla, R. (2014). liderando escuelas justas para la justicia social. Revista Internacional de Educación para la Justicia Social (RIEJS), 3(2), 13-32.

Rojas, S., Haya, I. y Lázaro-Visa, S. (2012). La voz del alumnado en la mejora escolar: Niños y niñas como investigadores en educación primaria. Revista de Educación, 359, 81-101.

Ros, I. (2009). La implicación del estudiante con la escuela. Revista de Psicodidáctica, 14(1), 79-92.

Rudduck, J. y Flutter, J. (2007). Cómo mejorar tu centro escolar dando la voz al alumnado. Madrid: Morata.

Sachs, S. B. (2002). Voices of reason: Adolescents talk about their futures over time. Westport, CT: Bergin \& Garvey.

San Fabián, J. L. (1994). Participar más y mejor. Cuadernos de Pedagogía, 224, 70-71.

Smyth, J. y Fasoli, L. (2007). Climbing over the rocks in the road to student engagement and learning in a challenging high school in Australia. Educational Research, 49, 273-95.

Solana, A. M. (2003). La participación de los alumnos en las clases de Educación Física: Una perspectiva cualitativa de sus comportamientos. Lecturas: Educación Física y Deportes, (67), 34-53.

Susinos, T. y Ceballos, N. (2012). Voz del alumnado y participación en la vida escolar: Apuntes para una cartografía de la voz del alumnado en la mejora educativa. Revista de Educación, 359, 24-44.

Thompson, P. (2009). Consulting secondary school pupils about their learning. Oxford Review of Education, 35(6), 671-687

Young, I.M. (2011). Responsabilidad por la justicia. Madrid: Morata.

\section{BY-NC-ND}

